\title{
RATIONAL MULTIPLIERS AND ANALYTICAL PROPERTIES OF THEIR TRANSFORMS
}

\author{
MICHAŁ WOJCIECHOWSKI \\ Institute of Mathematics, Polish Academy of Sciences \\ Śniadeckich 8, 00-950 Warszawa, Poland \\ E-mail:miwoj@impan.gov.pl
}

The results presented in this paper are concentrated around the analysis of translation invariant singular operators acting in the scale of $L^{p}$ spaces $(1 \leq p \leq \infty)$.

We study the operators acting on function spaces on abelian groups: $d$-dimensional euclidean spaces $\mathbb{R}^{d}$ and $d$-dimensional tori $\mathbb{T}^{d}$. A linear operator $T$ acting on the space $L^{p}(G)$ (where $G$ is one of the groups mentioned above) is called invariant iff $S_{a} T=T S_{a}$ for every $S_{a}$ where $S_{a}$ denotes the operator of shift by $a \in G$. The invariant operator $T: L^{p}(G) \rightarrow L^{p}(G)$ can be expressed in terms of the Fourier transform "^" and its inverse $" \vee "$ :

$$
T f=(m \widehat{f})^{\vee} .
$$

The function $m$ appearing in this formula is called a multiplier. Conversely, the operator $T_{m}$ corresponding to the multiplier $m$ is called a multiplier transform of $m$. The fundamental example of invariant operators are differential operators with constant coefficients. Multipliers which they generate are rational functions of several variables. These operators are our main object of study. They arise in the natural way in the theory of Sobolev spaces and they are the main analytical tools to study their properties. The behavior of such operators as $p \rightarrow 1$ (we are going to study the dependence of their $L^{p}$ norm on $\left.p \in[0, \infty]\right)$ is of particular interest. We stress that very often we deal with the singularities stronger that those described by the Calderón-Zygmund conditions (cf. [CZ], [St]).

The main class of operators which we study, as well as questions about their boundedness, arise from the theory of anisotropic Sobolev spaces. We shall present the multiplier theorems in this context, showing their natural motivations.

To describe more precisely this class we present briefly basic definitions concerning the Sobolev spaces (cf. e.g. [BBPW], [P]).

2000 Mathematics Subject Classification: Primary 42B15.

The paper is in final form and no version of it will be published elsewhere. 
The symbol $\mathbb{R}^{d}$ denotes $d$-dimensional euclidean space, $\mathbb{T}^{d}$ denotes $d$-dimensional torus and $\mathbb{Z}^{d}$ its dual group; $\mathbb{Z}_{+}^{d}$ denotes the set of elements of $\mathbb{Z}^{d}$ with non-negative coordinates. For $\alpha=\left(\alpha_{1}, \ldots, \alpha_{n}\right) \in \mathbb{Z}_{+}^{d}$ by $D^{\alpha} f$ we mean the distributional derivative $\partial_{x_{1}}^{\alpha_{1}} \ldots \partial_{x_{d}}^{\alpha_{d}} f$. We put $x^{\alpha}=x_{1}^{\alpha_{1}} \ldots x_{d}^{\alpha_{d}}$. A finite non-empty set $S \in \mathbb{Z}_{+}^{d}$ is called a smoothness iff it satisfies the following saturation condition: if $\alpha \in S$ and $\beta \leq \alpha$ then $\beta \in S(\alpha \leq \beta$ means that $\alpha_{j} \leq \beta_{j}$ for $\left.j=1,2, \ldots, d\right)$. Symbol \#S denotes the set of maximal elements of $S$ with respect to the relation " $\leq$ ". By $L_{S}^{p}\left(\mathbb{R}^{d}\right)$ we denote the Sobolev space of all functions $f: \mathbb{R}^{d} \rightarrow \mathbb{C}$ satisfying $D^{\alpha} f \in L^{p}\left(\mathbb{R}^{d}\right)$ for $\alpha \in S$. We define a norm in $L_{S}^{p}\left(\mathbb{R}^{d}\right)$ by the formula

$$
\|f\|_{p, S}=\sum_{\alpha \in S}\left\|D^{\alpha} f\right\|_{p} .
$$

Similarly we define the Sobolev space $L_{S}^{p}\left(\mathbb{T}^{d}\right)$ of functions on the torus. The Sobolev $L_{S}^{p}\left(\mathbb{R}^{d}\right)$ space has a very useful representation as a closed subspace of the space $L^{p}\left(\mathbb{R}^{d}, E\right)$ where $E$ is a euclidean space of dimension card $S$. We define this representation using the embedding

$$
\mathcal{J}: L_{S}^{p}\left(\mathbb{R}^{d}\right) \rightarrow L^{p}\left(\mathbb{R}^{d}, E\right)
$$

given by the formula

$$
\mathcal{J}(f)=\left(D^{\alpha} f\right)_{\alpha \in S} .
$$

$\mathcal{J}\left(L_{S}^{p}\left(\mathbb{R}^{d}\right)\right)$ is a closed subspace of the space $L^{p}\left(\mathbb{R}^{d}, E\right)$. For $p=2$ this is a closed subspace of a Hilbert space. We denote the corresponding orthogonal projection by

$$
\mathcal{R}_{S}: L^{p}\left(\mathbb{R}^{d}, E\right) \rightarrow \mathcal{J}\left(L_{S}^{p}\left(\mathbb{R}^{d}\right)\right) .
$$

Since $\mathcal{J}\left(L_{S}^{p}\left(\mathbb{R}^{d}\right)\right)$ is a translation invariant subspace, $R_{S}$ is an invariant operator. It is given by the Fourier multiplier $M_{S}$ :

$$
R_{S} f=\left(M_{S} \widehat{f}\right)^{\vee}
$$

where $\widehat{f}$ denotes the Fourier transform of $f$ and $g^{\vee}$ denotes the inverse transform. Since $\widehat{f}$ is a vector valued function, $M_{S}$ is a square matrix of size card $S$, whose elements are scalar valued Fourier multipliers. It is not difficult to find the concrete form of entries of $M_{S}$. We have

$$
M_{S}=\left[m_{\alpha, \beta}\right]_{\alpha, \beta \in S},
$$

where

$$
m_{\alpha, \beta}(x)=i^{|\alpha|-|\beta|} \cdot \frac{x^{\alpha+\beta}}{Q_{S}(x)},
$$

where

$$
Q_{S}(x)=\sum_{\alpha \in S} x^{2 \alpha}
$$

is the so called fundamental polynomial of the smoothness $S$. The knowledge of a form of the entries of $M_{S}$ allows us to extend the definition of $R_{S}$, which is defined at the moment only for $p=2$, to the spaces $L_{S}^{p}\left(\mathbb{R}^{d}\right)$ with other values of $p$. It is not difficult to check that the functions $m_{\alpha, \beta}$ satisfy the condition $\left(\mathrm{M}_{1}\right)$ of classical Marcinkiewicz's multiplier theorem (cf. [PS]). 
Definition. A function $m \in L^{\infty}\left(\mathbb{R}^{d}\right)$ satisfies condition $\left(\mathrm{M}_{k}\right)$ iff

$$
r_{1}^{\left|\alpha^{1}\right|-1} r_{2}^{\left|\alpha^{2}\right|-1} \ldots r_{d}^{\left|\alpha^{d}\right|-1} \int_{Y_{r}}\left|D^{\alpha} m(\xi)\right| d \xi<C,
$$

for every $\alpha=\left(\alpha^{1}, \ldots, \alpha^{d}\right) \in \mathbb{Z}_{+}^{d}$ such that $\left|\alpha^{j}\right| \leq k$ and $r=\left(r_{1}, r_{2}, \ldots, r_{d}\right) \in \mathbb{R}_{+}^{d}$, where $Y_{r}=\left\{\xi \in \mathbb{R}^{d}: r_{i}<\left|\xi_{i}\right|<2 r_{i}\right.$ for $\left.i=1,2, \ldots, d\right\}$.

Marcinkiewicz Theorem (cf. [S], VI.6.1). If $m \in L^{\infty}\left(\mathbb{R}^{d}\right)$ satisfies $\left(M_{1}\right)$ then $T_{m}$ is a bounded operator on $L^{p}\left(\mathbb{R}^{d}\right)$ for $1<p<\infty$.

In general $R_{S}$ is not bounded on $L^{1}\left(\mathbb{R}^{d}, E\right)$. The complete characterization of smoothnesses $S$ for which $R_{S}$ is $L^{1}$ bounded (i.e. $m_{\alpha, \beta}$ are the Fourier transforms of bounded measures for all $\alpha, \beta \in S$ ) is known. This holds iff $S$ contains exactly one maximal element, i.e. $S$ is of the form $S_{\alpha}=\left\{\gamma \in \mathbb{Z}_{+}^{d}: \gamma \leq \alpha\right\}$ (cf. [Si], [PS]).

A weak type $(1,1)$ of the operator could be considered as a substitute of $L^{1}$ boundedness. We say that the operator $T$ defined on dense subset of $L^{1}\left(\mathbb{R}^{d}, E\right)$ is of weak type $(1,1)$ if there exists $C>0$ such that

$$
\left|\left\{x \in \mathbb{R}^{d}:|T f(x)|_{E}>t\right\}\right|<C t^{-1}\|f\|_{1} .
$$

The importance of weak type $(1,1)$ is based on the theorem of Stein (in the invariant case) and Nikishin (in general) which says that every $f \in L^{1}\left(\mathbb{T}^{d}, E\right)$ has the image $T f$ defined almost everywhere if and only if $T$ is of weak type $(1,1)$.

It appears that for many smoothnesses the canonical projection $R_{S}$ is of weak type $(1,1)$. The most important examples are isotropic smoothnesses $S_{k}=\left\{\alpha \in \mathbb{Z}_{+}^{d}:|\alpha| \leq k\right\}$. It is easy to check this, because the corresponding multipliers $m_{\alpha, \beta}$ satisfy condition of Hörmander-Mihlin multiplier theorem (cf. $[\mathrm{H}]$ ).

Definition. A function $m \in L^{\infty}\left(\mathbb{R}^{d}\right)$ satisfies condition $\left(\mathrm{H}_{k}\right)$ iff

$$
r^{2|\alpha|-d} \int_{r<|x|<2 r}\left|D^{\alpha} m(\xi)\right|^{2} d \xi<C,
$$

for every $r>0$ and $\alpha \in \mathbb{Z}_{+}^{d}$ satisfying $|\alpha| \leq k$.

Hörmander-Minlin Theorem. If $m \in L^{\infty}\left(\mathbb{R}^{d}\right)$ satisfies condition $\mathrm{H}_{k}$ where $k$ is the smallest integer greater than $\frac{d}{2}$, then $T_{m}$ is of weak type $(1,1)$.

A consequence of a weak type $(1,1)$ of an operator is the estimation of the asymptotics of its $L^{p}$ norm which follows from the Marcinkiewicz interpolation theorem (cf. [Z], [St], $\S 1.4)$. If $T$ is of weak type $(1,1)$ and it is bounded on $L^{2}$ then there exists $C>0$ such that $\left\|T: L^{p} \rightarrow L^{p}\right\|<C(p-1)^{-1}$ for $p \rightarrow 1$. The first example of smoothness $S$ for which the canonical projection is not of weak type $(1,1)$ was based on this observation (cf. $[\mathrm{P}])$. A four-dimensional smoothness was constructed in $[\mathrm{P}]$ which was a tensor product of two two-dimensional isotropic smoothnesses and clearly it has the asymptotics of the form $(p-1)^{-2}$. This example was a base for the next two questions:

1) for which smoothnesses $S$ the canonical projection $R_{S}$ is of weak type $(1,1)$ ?

2) what is the asymptotics of $\left\|R_{S}: L^{p} \rightarrow L^{p}\right\|$ as $p \rightarrow 1$ for a fixed $d$-dimensional smoothness $S$ ? 
A partial answer to the first of these questions was given in [BBPW], where smoothnesses consisting of derivatives of order less than or equal to two, and two dimensional smoothnesses are considered.

THEOREM 1 ([BBWP]). For every two-dimensional smoothness the canonical Sobolev projection is of weak type $(1,1)$.

We call the smoothness $S$ non-degenerate iff all its maximal elements are of order greater than 1 ; we call $S$ reducible iff $x_{j}$ divides all the symbols of maximal elements of $S$ for some $j=1,2, \ldots, d$.

THEOREM 2 ([BBPW]). For every non-degenerate smoothness $S$ of order less than or equal to two one of the following holds true:

(i) all maximal elements of $S$ have order 1 and $S$ is an isotropic smoothness, so $R_{S}$ is of weak type $(1,1)$;

(ii) $S$ is reducible and $R_{S}$ is of weak type $(1,1)$;

(iii) $S$ is not reducible and all maximal elements of $S$ are of order two - then $R_{S}$ is of weak type (1,1) if and only if for every pair of different indices $i, j \in\{1,2, \ldots\}$ either $\frac{\partial^{2}}{\partial x_{j}^{2}} \in S$ and $\frac{\partial^{2}}{\partial x_{i}^{2}} \in S$, or $\frac{\partial^{2}}{\partial x_{i} \partial x_{j}} \in S$;

(iv) if $S$ is not reducible and the set $A$ consisting of all $i$ such that $\frac{\partial^{1}}{\partial x_{i}}$ is a maximal element of $S$ is nonempty, then $R_{S}$ is of weak type $(1,1)$ if and only if $\frac{\partial^{2}}{\partial x_{j}^{2}} \in S$ for every $j \notin A$.

Two different methods of proof of the lack of weak type $(1,1)$ can be used. One is based on the estimation from below of the asymptotics of the norm $\left\|R_{S}: L^{p} \rightarrow L^{p}\right\|$ as $p \rightarrow 1$ which contradicts Marcinkiewicz interpolation theorem. For some smoothnesses we were not able to find such an estimation and another method was developed which does not give a satisfactory information about the asymptotics of the $L^{p}$ norm. An example is a three-dimensional smoothness $S$ with $\# S=\{(2,0,0),(0,1,1)\}$. The corresponding multiplier which is not of weak type $(1,1)$ has the form $m(x, y, z)=\frac{x^{2} y z}{x^{4}+y^{2} z^{2}+y^{2}+z^{2}+1}$. Using the fact that "weak $(1,1)$ norm" of the Fourier multiplier does not change under composition with a linear transformation, and the closedness of the class of weak type $(1,1)$ operators with respect to the pointwise convergence, we derive that weak type $(1,1)$ of $T_{m}$ implies weak type $(1,1)$ of the operator $T_{n}$ where

$$
n(x, y, z)=\lim _{\lambda \rightarrow \infty} m_{\lambda}(x, y, z)=\lim _{\lambda \rightarrow \infty} m(\lambda x, \lambda y, \lambda z)=\frac{x^{4} y z}{x^{4}+y^{2} z^{2}} .
$$

Then, using the transference theorem for weak type $(1,1)$ operators (cf. [ABG], [Woz]), we get the that two-dimensional multiplier which is the restriction of $n$ to the plane $\{x=1\}$

$$
\phi(y, z)=\frac{y z}{1+y^{2} z^{2}}
$$

is of weak type $(1,1)$. This function depends on the product of variables. In [BBPW] it was shown that under some regularity conditions the corresponding multiplier transform is an integral operator with kernel depending on the product of variables. It is not difficult to show that such an operator may not be of weak type $(1,1)$. The proof of the fact that 
the kernel exists is sophisticated. In [W3] an alternative method of proof of the lack of weak type $(1,1)$ was presented. It can be applied to a much wider class of multipliers, since it does not use the algebraic properties of multipliers and it does not require passing through the Fourier transform.

Theorem 3 ([W3]). Let $\phi: \mathbb{R}^{d} \rightarrow \mathbb{C}$ be a continuous function. Suppose that there exist $a \in \mathbb{R}^{d}$, a sequence $\left(a_{j}\right)_{j=1}^{\infty} \subset \mathbb{R}^{d}$ and $C>0$ such that

$$
\begin{gathered}
\lim _{j \rightarrow \infty}\left|\left\langle a, a_{j}\right\rangle\right|=\infty, \\
\left|\phi\left(a_{j}\right)\right|>C \quad \text { for } j=1,2, \ldots, \\
\lim _{j \rightarrow \infty} \phi\left(x \pm a_{j}\right)=0 \quad \text { for } x \neq \lambda a .
\end{gathered}
$$

Then $T_{\phi}$ is not of weak type $(1,1)$.

Theorem 3 immediately implies that non-zero multipliers of the form $\phi(x, y)=g(x y)$, where $\lim _{|t| \rightarrow \infty} g(t)=0$, are not of weak type $(1,1)$.

One has to stress that the method of proof of Theorem 3 also does not give satisfactory information on the asymptotics of the $L^{p}$ norm.

The next result on which several estimations for anisotropic Sobolev spaces are based concerns the Fourier multipliers on Hardy spaces on product domains. We need some definitions to formulate it (cf. [ChF1], [ChF2], [F1], [F2]).

Denote by $R_{j}(j=1,2, \ldots, d)$ the multiplier transform of the function $x \mapsto \frac{x_{j}}{|x|}$ defined on $\mathbb{R}^{d}$. The operators $R_{j}$ are called the Riesz transforms. We put

$$
H^{p}\left(\mathbb{R}^{d}\right)=\left\{f \in L^{p}\left(\mathbb{R}^{d}\right): R_{j} f \in L^{p}\left(\mathbb{R}^{d}\right) \text { for } j=1,2, \ldots, d\right\} .
$$

We define the norm on $H^{p}\left(\mathbb{R}^{d}\right)$ by the formula

$$
\|f\|_{H^{p}}=\sum_{j=1}^{d}\left\|R_{j} f\right\|_{p} .
$$

Since the Riesz transform is a bounded operator on $L^{p}$ for $1<p<\infty$, we have $H^{p}\left(\mathbb{R}^{d}\right)=$ $L^{p}\left(\mathbb{R}^{d}\right)$ and the norms $\|\cdot\|_{p}$ and $\|\cdot\|_{H^{p}}$ are equivalent with the constant $(p-1)^{-1}$. The space $H^{1}$ differs from $L^{1}$, but for many applications it plays the role of a useful substitute of $L^{1}$.

Let $A \subset\{1,2, \ldots, d\}$. We define $h_{A}: \mathbb{R}^{d} \rightarrow \mathbb{R}$ by $h_{A}(x)=\prod_{j \in A} \operatorname{sgn} x_{j}$. Let $R_{A}$ denote the multiplier transform of the function $h_{A}$ i.e. $R_{A} f=\left(h_{A} \widehat{f}\right)^{\vee}$. We define the space $H^{p}(\mathbb{R} \times \ldots \times \mathbb{R})$ as the set of all those functions $f \in L^{p}\left(\mathbb{R}^{d}\right)$ for which $R_{A} f \in L^{p}\left(\mathbb{R}^{d}\right)$ for all $A \subset\{1,2, \ldots, d\}$. The norm in $H^{p}\left(\mathbb{R}^{d} \times \ldots \times \mathbb{R}^{d}\right)$ is given by

$$
\|f\|_{H^{p}\left(\mathbb{R}^{d} \times \ldots \times \mathbb{R}^{d}\right)}=\sum_{A \subset\{1,2, \ldots, d\}}\left\|R_{A} f\right\|_{p} .
$$

Since $\left\|R_{A}: L^{p} \rightarrow L^{p}\right\| \sim(p-1)^{-\# A}$ for $p \rightarrow 1$, we have $H^{p}(\mathbb{R} \times \ldots \times \mathbb{R})=L^{p}\left(\mathbb{R}^{d}\right)$ for $1<p<2$ and the norms $\|\cdot\|_{p}$ and $\|\cdot\|_{H^{p}\left(\mathbb{R}^{d} \times \ldots \times \mathbb{R}^{d}\right)}$ are equivalent with the constant $(p-1)^{-d}$.

THEOREM 4 ([W4]). If $m \in L^{\infty}\left(\mathbb{R}^{d}\right)$ satisfies condition $\left(M_{2}\right)$ then $T_{m}$ is a bounded operator on $H^{p}\left(\mathbb{R}^{d} \times \ldots \times \mathbb{R}^{d}\right)$. 
The proof of theorem 4 is based on the Littlewood-Paley theory, on the Stein theorem about multipliers on $H^{1}\left(\mathbb{R}^{d}\right)$ and on the Sobolev representation of function by means of its derivatives. We stress that the advanced theory of the atomic decomposition of $H^{1}$ functions on product domains (cf. [ChF1], [ChF2]) is not involved in the proof.

Theorem 4 could be applied also to the second question, i.e. to estimate the asymptotics of the norm of the canonical Sobolev projection on $L^{p}\left(\mathbb{R}^{d}, E\right)$ as $p \rightarrow 1$.

Corollary ([W5]). If $m \in L^{\infty}\left(\mathbb{R}^{d}\right)$ satisfies condition $\left(M_{2}\right)$ then there exists $C>0$ such that $\left\|T_{m}: L^{p} \rightarrow L^{p}\right\|<C(p-1)^{-d}$.

This is a good moment to compare Theorem 4 with the classical Marcinkiewicz theorem. The assumptions of Theorem 4 are stronger but one derives also a stronger result: there are multipliers $m$ satisfying the conditions of the Marcinkiewicz theorem for which $\left\|T_{m}: L^{p} \rightarrow L^{p}\right\|>C(p-1)^{-\frac{3}{2} d}$ (cf. [B]). In particular it follows that the Marcinkiewicz theorem alone is not sufficient to study the asymptotics of the norm of the canonical projection.

Since for any smoothness $S$ the entries of $M_{S}$ satisfy $\left(\mathrm{M}_{2}\right)$ we get

Theorem 5 ([W4]). For every d-dimensional smoothness $S$ there exists $C>0$ such that $\left\|R_{S}: L^{p} \rightarrow L^{p}\right\|<C(p-1)^{-d}$.

In [BBPW] an example was shown of $d$-dimensional smoothness $S$, given by $\# S=$ $\{(1,0,0, \ldots, 0),(0,1,1, \ldots, 1)\}$, for which $\left\|R_{S}: L^{p} \rightarrow L^{p}\right\|>C(p-1)^{-(d-1)}$. It remains an open question whether the exponent in the inequality from Theorem 5 could be replaced by $d-1$.

For every smoothness for which we know the precise asymptotics of the norm of the canonical Sobolev projection it is of the form $(p-1)^{-k}$ as $p \rightarrow 1$, where $k$ is an integer from the interval $1, \ldots, d$. We conjecture that this holds true for every $d$-dimensional smoothness. To support this conjecture we formulate the following result from [W1].

TheOREm 6 ([W1]). For every smoothness $S$ either $R_{S}$ is bounded on $L^{1}$ or $\| R_{S}$ : $L^{p} \rightarrow L^{p} \|>c(p-1)^{-1}$.

Theorem 6 has in fact a wider domain of applications than smoothnesses - it remains true for every multiplier $m$ for which there exists a sequence of balls $\left(B_{n}\right)$ with unbounded sequence of radii such that $\sup _{x, y \in B_{n}}|m(x)-m(y)| \rightarrow 0$ for $n \rightarrow \infty$ and the limit $\lim _{x \rightarrow \infty, x \in \cup B_{n}} m(x)$ does not exist.

The proof of Theorem 6 is based in one direction on the variant of the Boman method of representation of a monomial as a combination of monomials with coefficients being the Fourier transforms of bounded measures, and in the other direction on the Riesz product technique and martingale inequalities.

At the end we indicate briefly an application of Theorem 4 to interpolation of anisotropic Sobolev spaces. Namely we show that $L_{S}^{q}\left(\mathbb{R}^{d}\right)$ is an interpolation space for the couple $\left(L_{S}^{1}\left(\mathbb{R}^{d}\right), L_{S}^{p}\left(\mathbb{R}^{d}\right)\right)$ for $1<q<p<\infty$, where $S$ is an arbitrary smoothness. For suppose that a linear operator $T$ acting from $\mathcal{J}\left(L_{S}^{1}\left(\mathbb{R}^{d}\right)\right) \cap \mathcal{J}\left(L_{S}^{p}\left(\mathbb{R}^{d}\right)\right)$ to some Banach space $X$ is bounded in norms $L^{1}$ and $L^{p}$. Let $f \in \mathcal{J}\left(L_{S}^{q}\left(\mathbb{R}^{d}\right)\right)$. Then clearly $f \in H^{q}(\mathbb{R} \times$ $\ldots \times \mathbb{R}, E)$ where $\operatorname{dim} E=\# S$. By the known interpolation results for the Hardy spaces of 
product domains (cf. [ChF1]), there exist $g \in H^{1}(\mathbb{R} \times \ldots \times \mathbb{R}, E)$ and $h \in H^{p}(\mathbb{R} \times \ldots \times \mathbb{R}, E)$ such that $\|g\|_{H^{1}}+\|h\|_{H^{p}}<C \cdot\|f\|_{H^{q}}$ (more precise estimations for the $K$-functional are also available, cf. $[\mathrm{Mu}]$, but we use this just to show the idea). We have now $f=$ $\mathcal{R}_{S} f=\mathcal{R}_{S} g+\mathcal{R}_{S} h$ and, by Theorem $4, \mathcal{R}_{S}$ is bounded both on $H^{1}(\mathbb{R} \times \ldots \times \mathbb{R}, E)$ and on $H^{p}(\mathbb{R} \times \ldots \times \mathbb{R}, E)$. Thus $\left\|\mathcal{R}_{S} g\right\|_{1}+\left\|\mathcal{R}_{S} h\right\|_{p}<C_{1} \cdot\|f\|_{q}$, and we have $\|T f\|_{X} \lesssim$ $\|T g\|_{X}+\|T h\|_{X} \lesssim\|g\|_{1}+\|h\|_{p} \lesssim\|f\|_{q}$.

\section{References}

[ABG] N. Asmar, E. Berkson, and T. A. Gillespie, Convolution estimates and generalized de Leeuw theorems for multipliers of weak type (1,1), Canadian J. Math. 47 (1995), $225-245$.

[B] J. Bourgain, On the behavior of the constant in the Littlewood-Paley inequality, in: Geometric Aspects of Functional Analysis, Lecture Notes 1376, Springer Verlag, 1989.

[BBPW] E. Berkson, J. Bourgain, A. Pełczyński and M. Wojciechowski, Canonical projections of weak type (1,1), Mem. AMS 714 (2001), 1-75.

[CZ] A. P. Calderón and A. Zygmund, On the existence of certain singular integrals, Acta Math. 88 (1952), 88-139.

[ChF1] S.Y.A. Chang and R. Fefferman, The Calderón-Zygmund decomposition on product domains, Amer. J. Math. 104 (1982), 445-468.

[ChF2] S. Y. A. Chang and R. Fefferman, Some recent developments in Fourier analysis and $H^{p}$ theory on product domains, Bull. AMS 12 (1985), 1-43.

[FS] C. Fefferman and E. M. Stein, Hardy spaces of several variables, Acta Math. 129 (1972), 137-193.

[F1] R. Fefferman, Harmonic analysis on product spaces, Ann. of Math. 126 (1987), 109-130.

[F2] R. Fefferman, Some topics from harmonic analysis and partial differential equations, in: Essays on Fourier Analysis in Honor of Elias M. Stein, Princeton Univ. Press.

[Hö] L. Hörmander, The Analysis of Linear Partial Differential Operators I, Springer Verlag, 1983.

[Mu P. Mueller, Holomorphic martingales and interpolation, J. Anal. Math. 61 (1993), 327-337.

[P] A. Pełczyński, Boundedness of the canonical projection for Sobolev spaces generated by finite families of linear differential operators, in: Analysis at Urbana 1 (Proceedings of Special Year in Modern Analysis at the Univ. of Ill., 1986-87), London Math. Soc. Lecture Note Series 137, Cambridge Univ. Press, 1989, 395-415.

[PS] A. Pełczyński and K. Senator, On isomorphisms of anisotropic Sobolev spaces with "classical Banach spaces" and a Sobolev type embedding theorem, Studia Math. 84 (1986), 169-215.

[S] E. M. Stein, Singular Integrals and Differentiability Properties of Functions, Princeton Univ. Press, Princeton, 1970.

[W1] M. Wojciechowski, On the strong multiplier norm of rational functions in several variables, Ill. J. Math. 42 (1998), 582-600.

[W2] M. Wojciechowski, On the representation of function as a sum of derivatives, Compt. Rend. Acad. Sci. Paris 328 (1999), 303-306. 
[W3] M. Wojciechowski, A necessary condition for weak type $(1,1)$ of multiplier transforms, Canadian Math. Bull. 44 (2001), 121-125.

[W4] M. Wojciechowski, A Marcinkiewicz type multiplier theorem for $H^{1}$ spaces on product domains, Studia Math. 140 (2000), 273-287.

[Woz] K. Woźniakowski, A new proof of the restriction theorem for weak type $(1,1)$ multipliers on $\mathbb{R}^{n}$, Ill. J. Math. 40 (1996), 479-483. 